\title{
CAPILLARY BLOOD PRESSURE IN MAN. COMPARISON OF DIRECT AND INDIRECT METHODS OF MEASUREMENT. ${ }^{1}$
}

\author{
By L. W. EICHNA AND JAMES BORDLEY, III \\ (From the Department of Medicine, The Johns Hopkins University School of Medicine, \\ Baltimore)
}

(Received for publication July 10, 1939)

In the course of studies of the peripheral circulation in hypertensive subjects it became desirable to make measurements of the capillary blood pressure. Previous studies by others (1, $2,3)$ had indicated that the capillary blood pressure is at approximately the same level in normal and hypertensive subjects. The accuracy of the indirect methods of measurement employed in these earlier studies has never been actually established. Indeed there is reason to suspect their reliability (4). It seemed necessary, therefore, before attempting to determine capillary blood pressure in hypertensive subjects, to analyze critically some of the available methods of measurement, in order to select one which would yield trustworthy results. Two methods were chosen for analysis: (1) the direct microinjection method of Landis (5), and (2) the indirect, pressurecapsule method of Danzer and Hooker (6).

Heretofore, no attempt has been made to compare in the same individual the results obtained by the direct and indirect methods. The present investigation has led to the conclusion that the two methods do not give comparable results, and that, of the two, only the direct method gives accurate readings.

\section{METHODS}

Because of their size, accessibility and position parallel to the skin surface, the capillaries in the nail folds are, in man, the most suitable for blood pressure determinations. All of the following observations were made upon capillaries in the nail folds of the fingers of quiet, recumbent subjects with normal systemic venous pressures. The capillary bed was always at the level of the heart, i.e., 2 to $5 \mathrm{~cm}$. below the level of the anterior chest wall at the third intercostal space. The observations were made in a quiet, warm room, the temperature of which was usually $24^{\circ}$ to $26^{\circ} \mathrm{C}$. In none of the experiments was the room temperature higher than $28^{\circ} \mathrm{C}$. or lower than $22^{\circ}$ C. Digital skin temperature varied from sub-

1 Supported by a grant from The Commonwealth Fund for the study of essential hypertension. ject to subject but was fairly constant in any single experiment. The temperature of the finger tip was usually between $28^{\circ}$ and $31^{\circ} \mathrm{C}$, and always between $25^{\circ}$ and $33^{\circ} \mathrm{C}$.

\section{Indirect method (Danzer and Hooker)}

The apparatus of Danzer and Hooker (6) consists essentially of a pressure capsule, with a floor of transparent glycerin-soaked goldbeaters skin, connected by pressure tubing with a mercury manometer and a pressure changing device. The capillaries are observed directly through the capsule, the membrane of which rests upon the nail fold. In making a reading, the pressure in the capsule is raised until blood flow stops in the capillary which has been selected for observation. The capsule pressure is then slowly reduced until onward capillary flow returns, usually very abruptly. The manometer reading at this point is taken to represent the capillary blood pressure.

This method is technically easy, and permits determinations of pressure in several capillaries at one time. There are, however, the following serious theoretical objections to it: (1) Capillary blood flow is altered from the normal in making a reading. (2) It is impossible to estimate how much of the pressure of the capsule is taken up by the overlying tissue before being transmitted to the capillaries. (3) Pressure is made upon other minute vessels (arterioles, venules) as well as upon the capillaries. This external pressure may alter the blood pressure in these other vessels and thereby affect the capillary blood pressure. (4) Since but one reading is obtained for each capillary, the gradient of pressure in the capillary cannot be determined.

\section{Direct method (Landis)}

With but few modifications, the method and apparatus used were those devised by Landis (5). A pyrex-glass micropipette (orifice 6 to $10 \mu$ in diameter) is fastened in a micromanipulator ${ }^{2}$ by means of which the micropipette may be moved in any desired direction. A smallbored flexible brass tube connects the micropipette with

2 The Chambers micromanipulator used by Landis was replaced by one designed for Dr. A. N. Richards by Mr. Carol Kelly, machinist at the University of Pennsylvania Medical School. This manipulator affords, in addition to lateral and vertical movements, a straight longitudinal movement in the direction of the long axis of the micropipette. 
a manometer system in which the pressure may be changed at will by means of a plunger driven by a fine thumbscrew. The micropipette, brass tube and manometer system are filled with Ringer's solution ( $\mathrm{pH} 7.3$ to 7.4), with heparin ( $3 \mathrm{mgm}$. per $100 \mathrm{cc}$. of solution) added as an anticoagulant.

In determining the capillary blood pressure, the tip of the micropipette is carefully introduced into the lumen of a capillary loop at the desired location. Since the initial pressure of the solution in the micropipette is only 2 to $3 \mathrm{~mm}$. $\mathrm{Hg}$, the entry of the micropipette into the capillary is signaled by a rush of blood into the pipette tip. As soon as this occurs, the pressure in the micropipette is quickly raised, forcing most of the red blood cells back into the capillary. By further adjustment of the pressure, a state of equilibrium is reached in which a few red blood cells remain in the extreme tip of the micropipette, oscillating back and forth with each heart beat, but manifesting no tendency to move progressively either into or out of the tip. The manometer reading at this equilibrium point, after corrections have been made for the capillarity of the manometer and for the difference in level between the base of the manometer and the level of the nail fold, represents the capillary blood pressure. The balance between manometer pressure and capillary pressure at the equilibrium point is a very delicate one: changes of 2 to $3 \mathrm{~mm}$. $\mathrm{Hg}$ in manometer pressure suffice to establish progressive movement of the red cells either into or out of the micropipette. Throughout this entire procedure the capillary blood flow should not be changed from the normal, swiftly-flowing stream. If the blood flow appears to be obstructed or otherwise altered, the pressure readings are considered unacceptable. It has been necessary to discard a number of readings on this account.

In order to facilitate the passage of the fragile micropipette through the skin, the most superficial layers of the epidermis of the nail fold were always cut away with a keen razor blade. Only non-living tissue was removed; and pain, bleeding and tissue damage were avoided. This procedure was carried out approximately an hour before determinations of pressure were made. The nail folds were prepared in this manner before both the direct and indirect measurements.

The direct method has several shortcomings: (1) The technical difficulties are formidable. (2) The insertion of the micropipette frequently causes such changes in capillary blood flow (stasis) that acceptable pressure readings cannot be obtained. (3) Determinations of pressure can be made on only one capillary at a time; hence, relatively few capillaries can be studied in the course of a single experiment.

\section{The critical test}

In order to evaluate the reliability of the two foregoing methods, both have been subjected to what may be called a critical test. This test is carried out in the following manner: A pneumatic cuff (width $14 \mathrm{~cm}$.) is placed loosely about the arm above the elbow. After several determinations of capillary pressure have been made, the venous pressure distal to the cuff is raised by inflating the cuff to a pressure below diastolic arterial pressure. During the ensuing period of elevated venous pressure, the determinations of capillary pressure are repeated. The cuff is then deflated, allowing the venous pressure to fall to its original level, and the capillary pressure determinations are again repeated.

The following considerations led to the use of this type of critical test. When a pneumatic cuff encircling the upper arm is inflated, the venous pressure distal to the cuff rises and within one minute very closely approximates the pressure within the cuff (Lewis (7)), provided the cuffpressure does not exceed diastolic arterial pressure. Under these circumstances, in spite of the increased venous pressure, the blood flow in the capillaries of the nail fold can be seen to persist steadily onward, though usually at a slower rate. This persistence of capillary blood flow indicates that the capillary blood pressure has risen to exceed the elevated venous pressure. Landis (5), in testing the accuracy of the direct method, made use of this principle, and observed that the directly-determined capillary pressure rose to exceed the cuff-pressure. It is believed, therefore, that a reliable method for the determination of capillary blood pressure should show that capillary pressure exceeds venous pressure so long as blood is flowing through the capillaries into the veins.

\section{RESULTS}

\section{Relation of capillary pressure to occluding cuff-pressure}

The charts indicate the type of results obtained when each of the two methods was subjected to the critical test. The capillaries were studied by each of the methods during the same observation period and hence under relatively uniform conditions. The method which was employed first is charted first.

Figures 1 to 4 exemplify the difference in results obtained by the two methods. The indirect method fails to detect any significant change in capillary blood pressure when the venous pressure rises. On the other hand, the direct method in- 


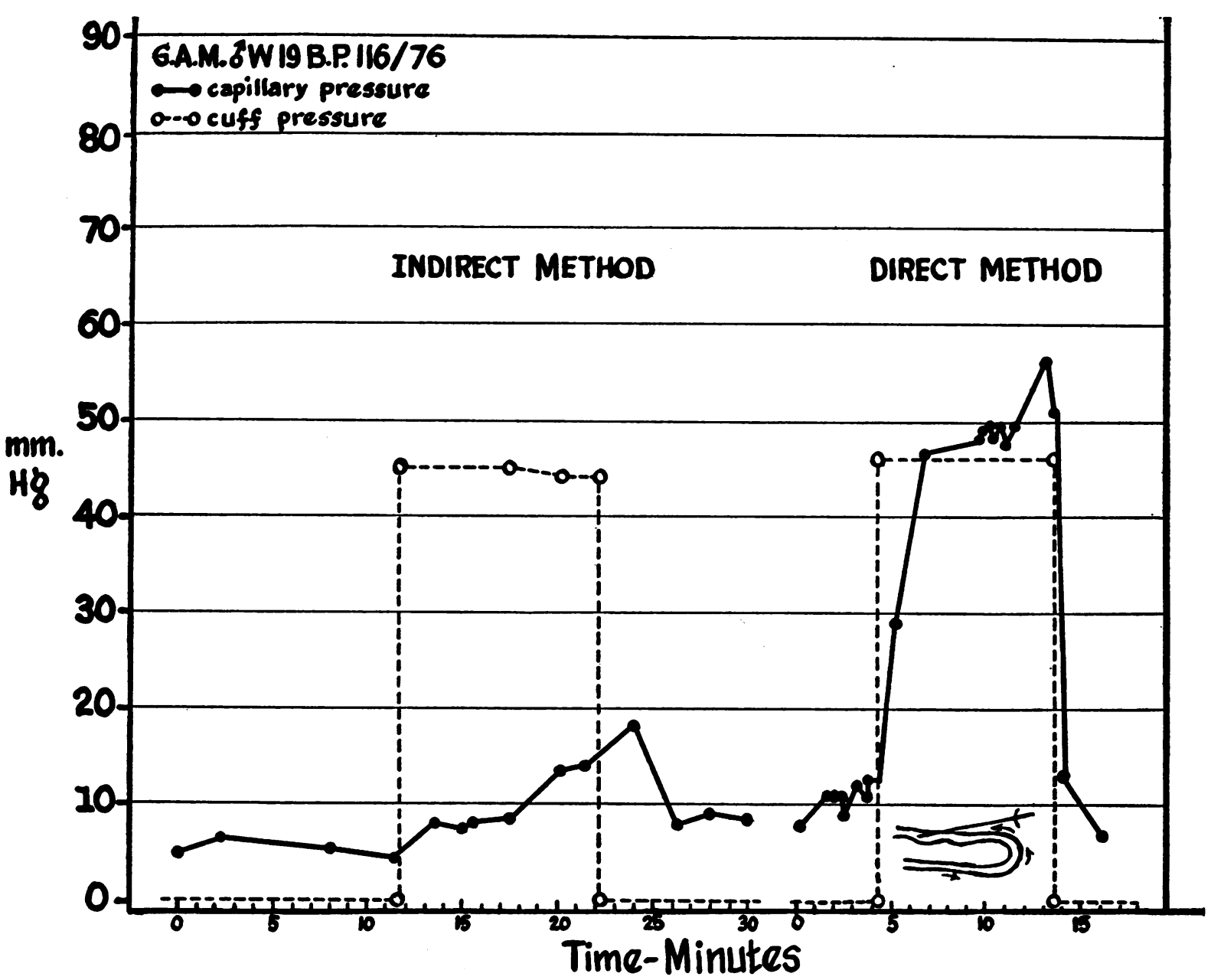

Fig. 1. Response of Capillary Blood Pressure to Increased Venous Pressure in a Subject with Normal Blood Pressure

Indirect method: no significant rise. Direct method (venous limb) : prompt rise to exceed cuff pressure.

All charts are similarly plotted. The ordinates represent millimeters of mercury for both cuff and capillary pressures; the abscissae, time in minutes. The solid line indicates the observed capillary pressure reading by both the direct and indirect methods. The dotted line is cuff pressure. In the diagram of the capillary, the straight line represents the location of the pipette in the capillary loop; the arrows, the direction of capillary blood flow. The first line of the key in the upper left hand corner of the charts indicates, in order, the subject's initials, sex, color, age, and blood pressure. The method which was employed first is charted first.

variably records a prompt rise in capillary blood pressure, the elevation persisting throughout the entire period that the cuff is inflated.

Observations by the direct method were made upon 31 different capillaries in 18 subjects; 9 with normal, 7 with high and 2 with low arterial blood pressure. In each of these capillaries a prompt rise in blood pressure was always recorded during the critical test. In 21 capillaries the pressure rose to exceed the cuff-pressure. In the remain- ing 10 (to be discussed subsequently), the capillary pressure rose promptly but failed by 2 to 5 $\mathrm{mm}$. $\mathrm{Hg}$, to reach the level of the cuff-pressure. In well over 200 capillaries studied by the indirect method, the capillary pressure readings during the critical test showed either no change, or, at the most, only slight and inconstant rises above the initial level. In 9 experiments a single capillary was studied by each of the two methods during the critical test, always with the above-mentioned 


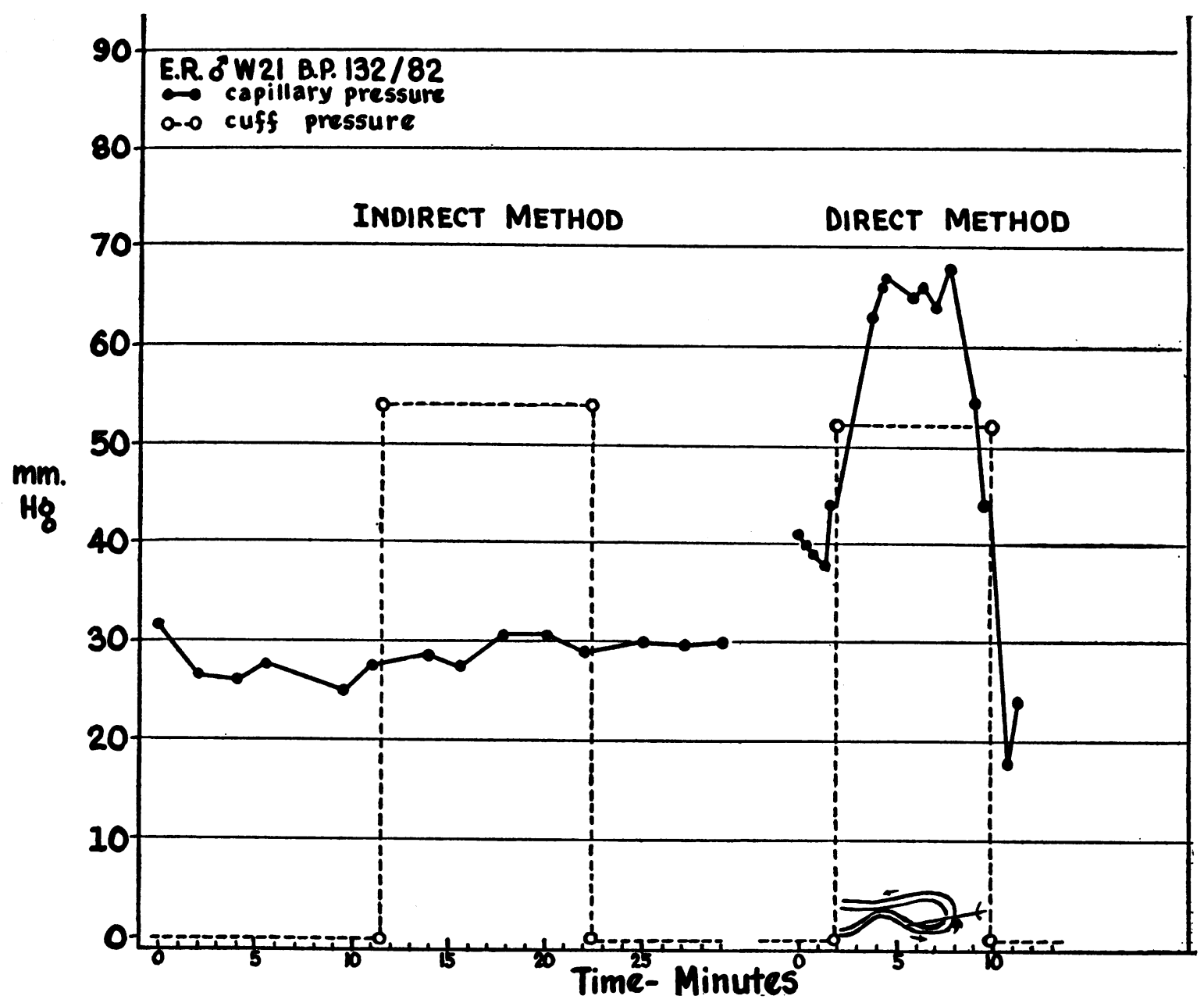

Fig. 2. Response of Capillary Blood Pressure to Increased Venous Pressure in a Subject with Normal BLOOD Pressure

Indirect method: no change whatever. Direct method (arteriolar limb): quick response to exceed cuff pressure. Capillary stasis at the time of the last four readings probably accounts for their low level.

difference in result. The capillary pressure response typical for each method was obtained at all cuff-pressures between the limits of 36 and 90 $\mathrm{mm}$. Hg. The higher cuff-pressures were employed in hypertensive subjects, in one of whom a direct capillary pressure reading of $102 \mathrm{~mm}$. $\mathrm{Hg}$ was obtained at a cuff-pressure of $90 \mathrm{~mm}$. $\mathrm{Hg}$.

Since the capillary blood flow persisted steadily onward during the period of elevated venous pressure in all of the foregoing experiments, it may be assumed that the capillary blood pressure actually exceeded the venous pressure. Therefore, it has been concluded, that, whereas the direct method yields accurate estimates of capillary blood pressure, the indirect method fails to do so.
The direct method has also shown that rapid fluctuations in capillary blood pressure may occur spontaneously (see Figures 4 and $6 A$ ). These are not detected by the indirect method.

\section{Relation of venous pressure in the hand to} occluding cuff-pressure

In the earlier experiments with the critical test it was assumed that the pressure in the occluding cuff accurately represented the pressure in the veins of the hand. It became necessary to inquire whether this was a correct assumption when observations (see above) were recorded in which the directly-determined capillary pressure, although rising promptly with cuff inflation, failed 
by 2 to $5 \mathrm{~mm}$. $\mathrm{Hg}$ to reach cuff-pressure (Figure 5). In checking over the set-up of the experiments, it was noted that the nail fold had usually been at a level materially higher than that of the most dependent portion of the occluding cuff. The question arose whether this difference in level between the hand and the cuff might lead to a discrepancy between the pressure in the veins of the hand and that in the cuff. To answer this question the pressure was determined in the veins of the dorsum of the hand ${ }^{3}$ at various occluding cuff-pressures, while the relation of the level of the hand to the level of the cuff was being changed through a wide range. Table I gives the results

$3 \mathrm{By}$ the direct method of Moritz and v. Tabora.
TABLE I

Relation of venous pressure in the hand to cuff-pressure during change of level of hand

\begin{tabular}{|c|c|c|c|}
\hline $\begin{array}{l}\text { Relation of hand } \\
\text { vein to most } \\
\text { dependent part } \\
\text { of cuff }\end{array}$ & $\underset{\text { pressure }}{\text { Cuff }}$ & $\begin{array}{l}\text { Venous } \\
\text { pressure }\end{array}$ & Difference \\
\hline $\begin{array}{r}13.0 \mathrm{~cm} \text {. above... } \\
9.7 \mathrm{~cm} \text {. above... } \\
5.4 \mathrm{~cm} \text {. above... } \\
1.6 \mathrm{~cm} \text {. above... } \\
1.7 \mathrm{~cm} \text {. above... } \\
1.7 \mathrm{~cm} \text {. above... } \\
1.7 \mathrm{~cm} \text {. above... } \\
3.5 \mathrm{~cm} \text {. below... } \\
3.5 \mathrm{~cm} \text {. below... }\end{array}$ & $\begin{array}{c}m m . H 8 \\
60 \\
60 \\
60 \\
60 \\
60 \\
52 \\
34 \\
34 \\
52\end{array}$ & $\begin{array}{c}m m . H^{*} \\
54.3 \\
56.1 \\
57.9 \\
61.3 \\
62.5 \\
54.3 \\
35.5 \\
42.1 \\
59.2\end{array}$ & $\begin{array}{c}m m . H_{8} \\
-5.7 \\
-3.9 \\
-2.1 \\
+1.3 \\
+2.5 \\
+2.3 \\
+1.5 \\
+8.1 \\
+7.2\end{array}$ \\
\hline
\end{tabular}

* Actually determined in mm. of isotonic salt solution and converted to $\mathrm{mm}$. $\mathrm{Hg}$.

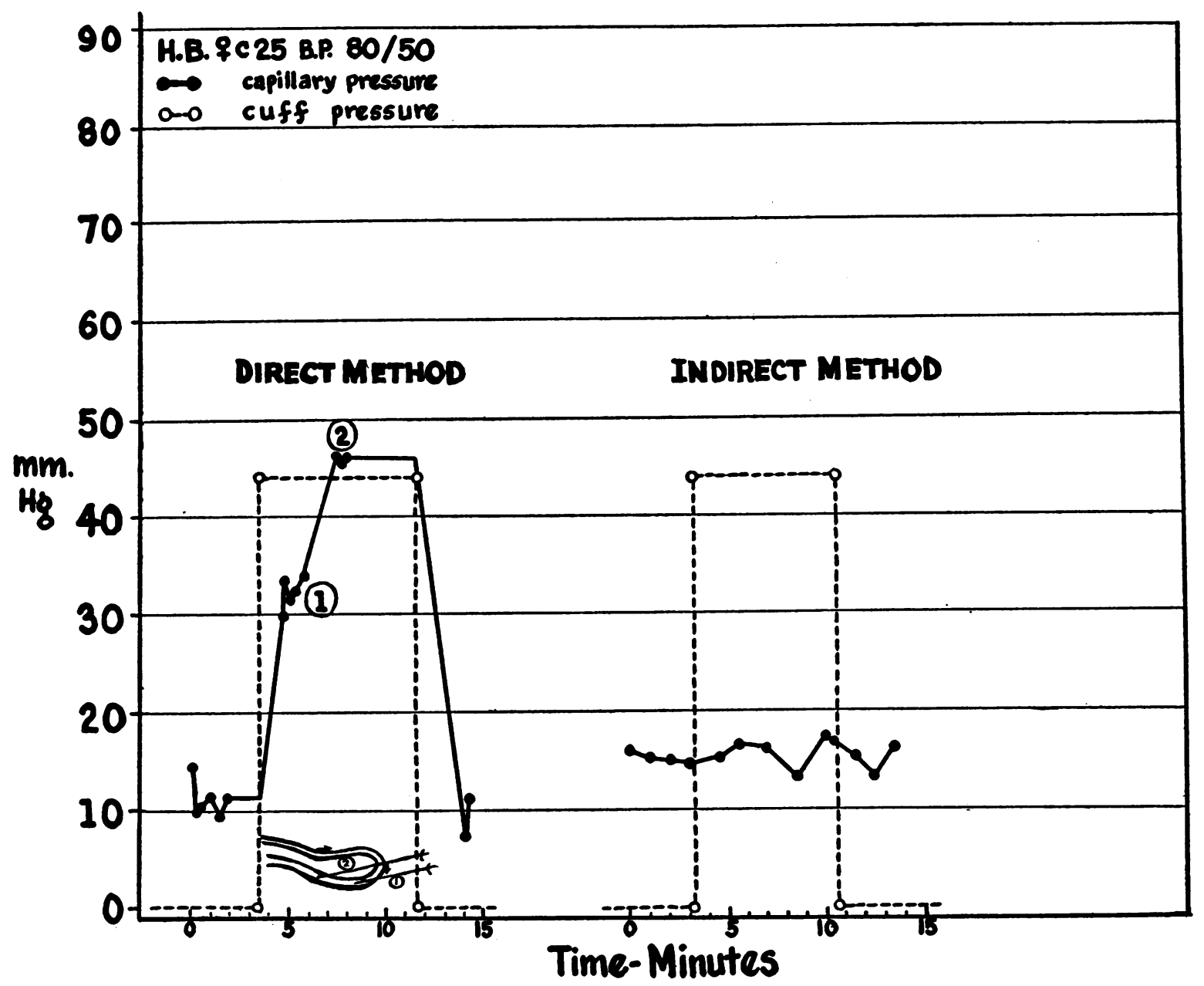

Fig. 3. Response of Capillary Blood Pressure to Increased Venous Pressure in a Subject with Arterial Hypotension

Direct method (venous limb) : capillary pressure finally exceeded cuff pressure. During readings at (1) flow was obstructed by the pipette. Indirect method: capillary pressure unaltered. 


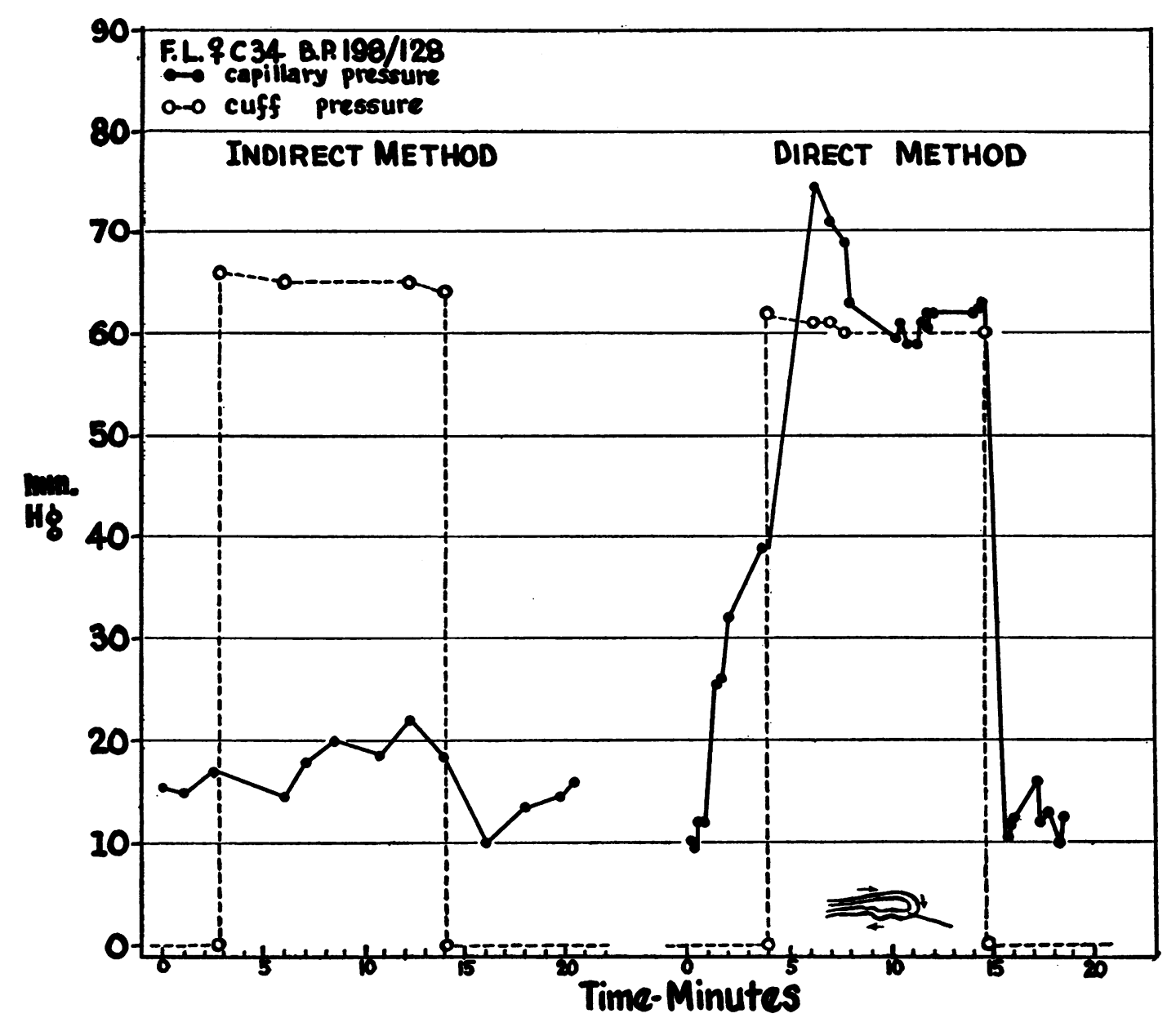

Fig. 4. Response of Capillary Blood Pressure to Increased Venous Pressure in a Subject with ArTerial Hypertension

Indirect method: no significant change. Direct method (summit of loop) : prompt response to exceed cuff pressure. Note spontaneous rise in capillary pressure in the period before cuff inflation.

obtained in a typical experiment. Similar results were obtained in six additional experiments.

These experiments show that venous pressure is less than cuff-pressure when the hand vein is at a level above that of the most dependent portion of the cuff. The difference between the two pressures can be accounted for by the hydrostatic relation between the level of the hand and that of the cuff.

During the critical test the nail fold was usually 9 to $12 \mathrm{~cm}$. above the level of the most dependent portion of the occluding cuff. This indicates that the venous pressure in the hand was usually 3 to $6 \mathrm{~mm}$. Hg less than cuff-pressure. Direct venous pressure measurements in several experiments have confirmed this occlusion.

Having established these relationships, it was concluded that capillary pressure during the critical test had probably exceeded the venous pressure in the hand in all experiments, even in those in which the cuff-pressure had not been reached. Figure $6 \mathrm{~A}$ gives evidence of the correctness of this conclusion. In this observation the directlydetermined capillary pressure failed by 1.5 to 4.5 $\mathrm{mm}$. $\mathrm{Hg}$ to reach cuff-pressure but exceeded the actually measured venous pressure (horizontal arrow) by 6 to $1.5 \mathrm{~mm}$. $\mathrm{Hg}$.

Unless these relationships are borne in mind, 
erroneous conclusions may also be reached in interpreting those observations in which, during the critical test, the capillary pressure exceeded the cuff pressure. Figure $6 B$ is an example of this. It shows that, whereas the capillary pressure was higher than cuff pressure by 0.5 to $8 \mathrm{~mm}$. $\mathrm{Hg}$, the measured venous pressure was exceeded by 4 to $13 \mathrm{~mm}$. $\mathrm{Hg}$.

\section{Subsidiary experiments with the indirect, pressure-capsule method}

The possibility was considered that the apparent inadequacy of the indirect method to measure changes in capillary pressure might be attributed to some local peculiarity of the nail fold capillaries. Consequently, attempts were made to apply the pressure-capsule to other skin areas in which venous pressure could be altered as required for the critical test. Most of the skin areas of the extremities were found to be either too uneven or too yielding to permit proper application and use of the capsule. The skin overlying the flat antero-medial surface of the tibia afforded an excellent site, but here the capillary pressure readings were vitiated by the spontaneous intermissions in capillary blood flow which are so frequent in this area (8).

Changing the position of the nail fold in rela-

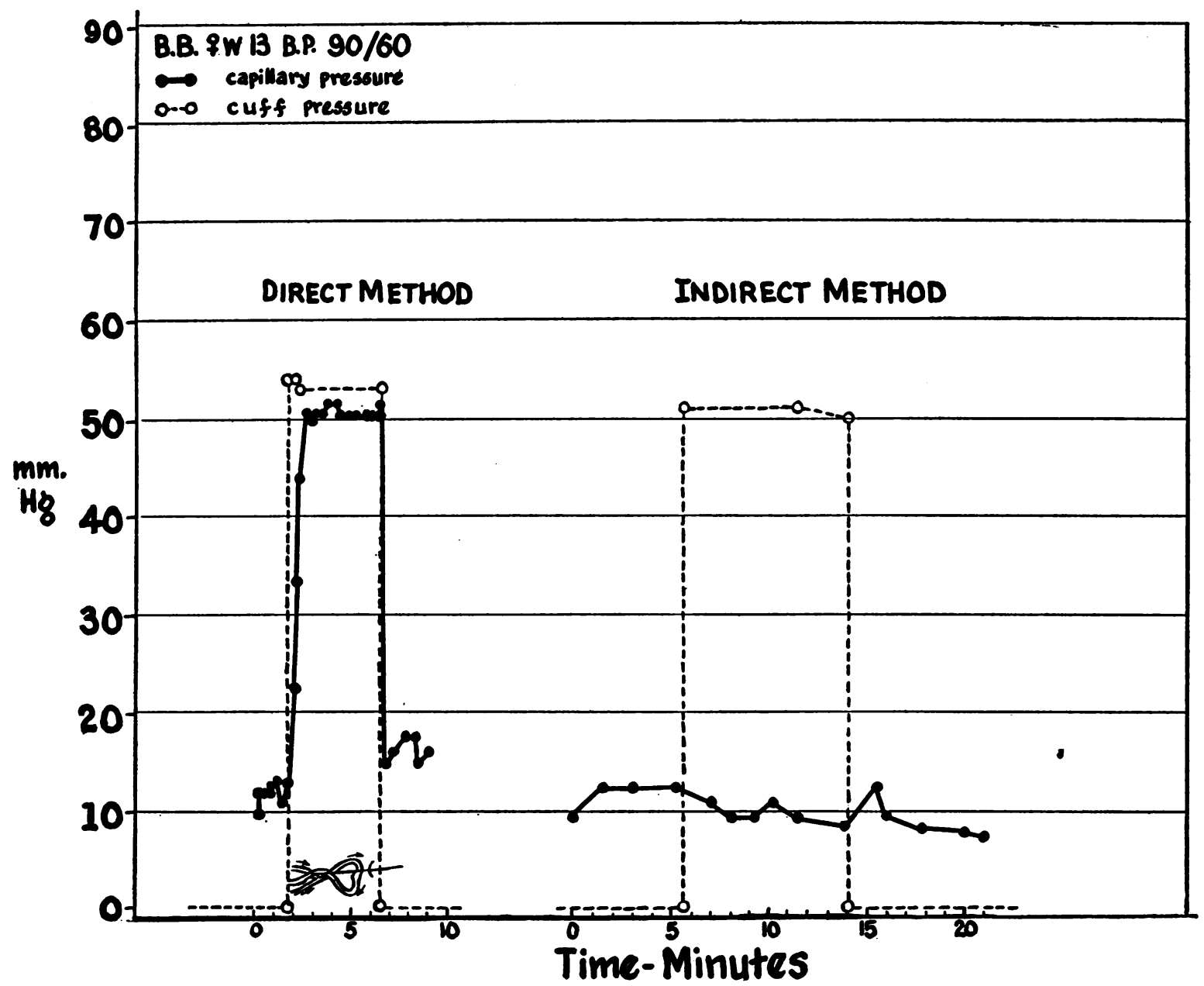

Fig. 5. Response of Capillary Blood Pressure to In creased Venous Pressure in a Diabetic with Normal Blood Pressure

Direct method (venous limb) : capillary pressure rises and falls promptly but fails to reach cuff pressure by 1.5 to $3.0 \mathrm{~mm}$. Hg. Indirect method: unchanged capillary pressure. 


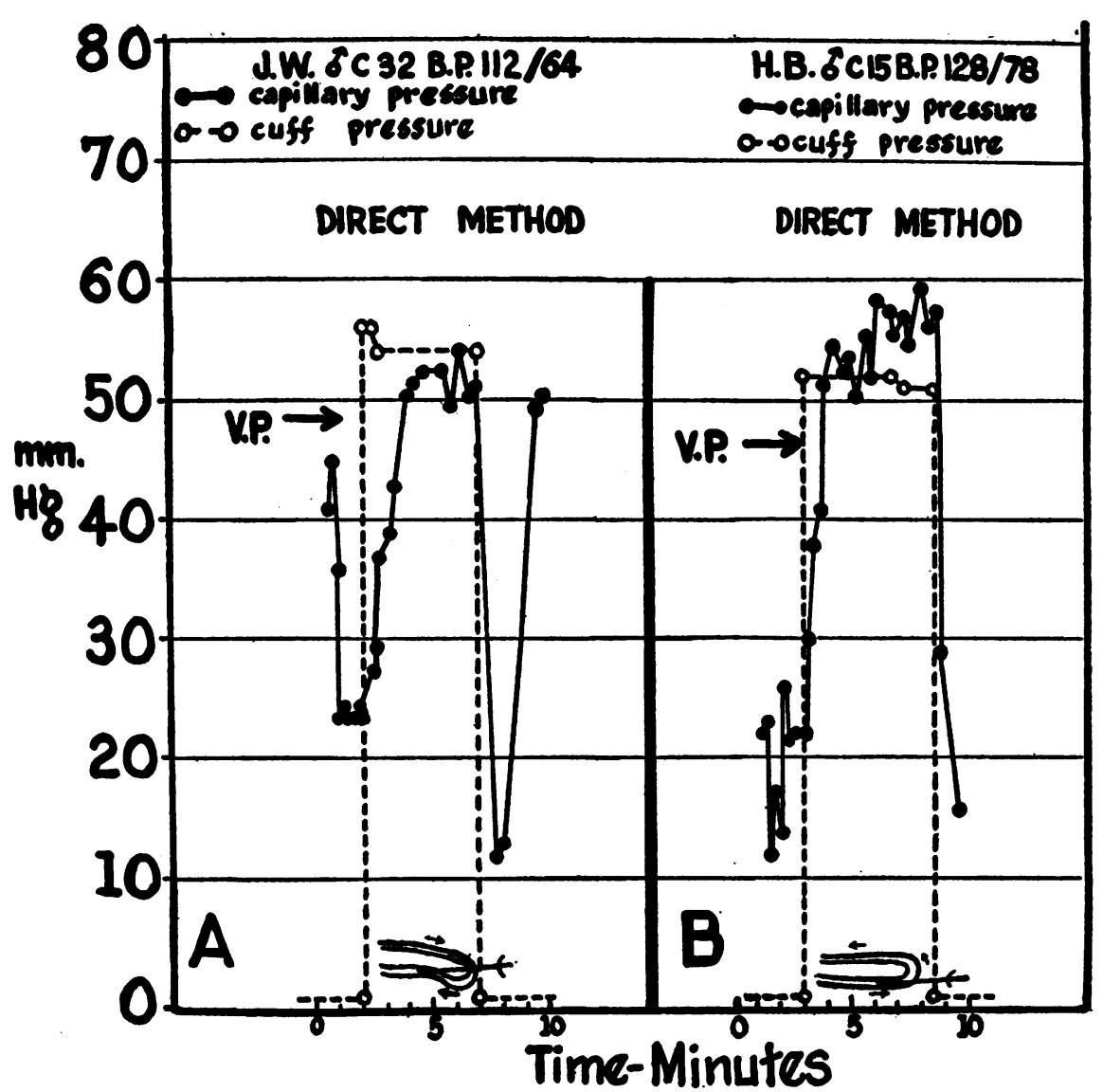

Fig. 6 A. Response of Capillary Blood Pressure to Increased Venous Pressure in a Subject with Normal Blood Pressure

Direct method (venous limb): during critical test capillary pressure rises and falls promptly but fails to reach cuff pressure. However capillary pressure exceeds the actual venous pressure (large arrow). Note wide spontaneous fluctuations before and after cuff inflation.

Fig. 6 B. Response of Capillary Blood Pressure to Increased Venous Pressure in a Subject with Normal Blood Pressure

Direct method (arteriolar limb) : capillary pressure exceeds the cuff-pressure but not by as great a margin as it exceeds the measured venous pressure (horizontal arrow).

tion to the level of the heart was found to produce no constant change in the indirectly-determined capillary pressure. In a typical experiment the readings were within the same range whether the nail fold was $18 \mathrm{~cm}$. below or $1 \mathrm{~cm}$. above the level of the sternum. Landis (5) has shown that the directly-determined blood pressure in the capillaries of the nail fold rises when the hand is lowered, and falls when the hand is raised.

In a patient with a mediastinal tumor producing obstruction of the superior vena cava, the in- direct method gave capillary pressure readings which were lower than the venous pressure in the hand. The venous pressure was $18.1 \mathrm{~mm}$. $\mathrm{Hg}$, while the average pressure in 11 nail fold capillaries was $11 \mathrm{~mm}$. $\mathrm{Hg}$ (range of readings 7.5 to $18.0 \mathrm{~mm}$. $\mathrm{Hg}$ ).

The failure of the pressure-capsule method to detect changes in capillary blood pressure during periods of increased venous pressure was further investigated by carrying out the critical test under the following experimental conditions. 
(1) Anesthetization of the nail fold. This was accomplished by two methods: (a) by injecting a 2.5 per cent solution of procaine into the skin 0.5 to $1 \mathrm{~cm}$. proximal to the nail fold, (b) by injecting a 2.5 per cent solution of procaine into the ulnar nerve at the elbow. In the latter case the capillaries of the anesthetized fifth finger were used for pressure determinations. With each type of anesthetization one experiment was performed. In both experiments the indirect method failed to detect a significant rise in capillary blood pressure during the critical test. The directly-determined capillary blood pressure rose promptly during the critical test in the one experiment in which this was performed following ulnar nerve block (this subject had Raynaud's disease).

(2) Procedures designed to relax the tone of the minute vessels. Two procedures were used to relax vascular tone prior to carrying out the critical test: ( $a$ ) generalized heating of the body until digital skin temperature had reached maximum values, and (b) puncturing 1:1000 histamine into the skin at several points about $0.5 \mathrm{~cm}$. proximal to the nail fold. In the latter case the capillary pressure estimations were made while the nail fold was involved in the red flare of the histamine reaction. Even after the vascular tone had been relaxed by the foregoing methods, the indirectly-determined capillary pressure failed to rise appreciably during the critical test.

These subsidiary experiments give additional grounds for doubting the aceuracy of the pressure-capsule method.

\section{DISCUSSION}

Although it has been concluded that the microinjection method yields reliable measurements of capillary blood pressure, it is realized that the absolute accuracy of the method depends upon the extent to which normal capillary physiology is altered by the insertion of the micropipette. It has been reassuring to find that, under favorable circumstances, piercing the capillary causes no visible change in the capillary blood flow. Under such conditions it is believed that the values obtained represent true capillary blood pressure.

It is noteworthy that under conditions of normal venous pressure the indirect method yields figures for capillary blood pressure which are of the same order of magnitude as those obtained by the microinjection method. The present experiments indicate that this agreement may be fortuitous, for the results obtained by the two methods fail to correspond when the venous (and capillary) pressure is raised. In the latter event, the pressure-capsule method yields values which are far too low to be acceptable. Since this method appears to be unreliable under conditions of changing capillary pressure, there is no assurance that it is reliable under any circumstances. The other methods for measuring capillary blood pressure, which are based upon similar principles, may likewise be untrustworthy. No satisfactory explanation for the inadequacy of the indirect, pressure-capsule method has been adduced.

In view of the questionable accuracy of the indirect methods heretofore employed, the subject of capillary blood pressure in human pathologic states deserves reinvestigation by direct methods. This has already been done by Landis $(9,10)$ in certain vasospastic conditions.

\section{SUMMARY AND CONCLUSIONS}

1. Two methods of measuring capillary blood pressure in man were compared: $(a)$ the indirect pressure-capsule method of Danzer and Hooker, and $(b)$ the direct microinjection method of Landis. The comparison was made both at normal and at elevated levels of venous pressure.

2. When a cuff encircling the upper arm was inflated to pressures below diastolic arterial pressure, the venous pressure in the hand rose. The extent of the rise was influenced by the difference in level between the cuff and the hand.

3. The capillary blood pressure in the nail fold, recorded by the direct method, was found always to exceed the venous pressure in the hand.

4. The capillary blood pressure in the nail fold, recorded by the indirect method, failed to show any correlation with the venous pressure in the hand.

5. It has been concluded that of the two methods only the direct one affords an accurate measurement of capillary blood pressure.

Drs. William B. Sherman and Max H. Grow participated in the preliminary studies of the pressure-capsule method. Some of the information which they helped to 
obtain has been included in this report. The authors are also indebted to Mr. William A. Oktavec, Jr., for his constant and very helpful assistance.

\section{BIBLIOGRAPHY}

1. Boas, E. P., and Frant, S., Capillary blood pressure in arterial hypertension. Arch. Int. Med., 1922, $30,40$.

2. Ellis, L. B., and Weiss, S., Measurement of capillary pressure under natural conditions and after arteriolar dilatation in normal subjects and in patients with arterial hypertension and with arteriosclerosis. J. Clin. Invest., 1930, 8, 47.

3. Mufson, I., Study of capillary pressure in nephritis and hypertension. Am. J. Med. Sc., 1932, 183, 632.

4. Landis, E. M., Capillary pressure and capillary permeability Physiol. Rev., 1934, 14, 404.
5. Landis, E. M., Microinjection studies of capillary blood pressure in human skin. Heart, 1930, 15, 209.

6. Danzer, C. S., and Hooker, D. R., Determination of capillary blood pressure in man with the microcapillary tonometer. Amer. Jour. Physiol., 1920, $52,136$.

7. Lewis, T., Force exerted by minute vessels of the skin in contracting. Heart, 1924, 11, 109.

8. Bordley, J., III, Grow, M. H., and Sherman, W. B., Intermittent blood flow in capillaries of human skin. Bull. Johns Hopkins Hosp., 1938, 62, 1.

9. Lewis, T., and Landis, E. M., Observations upon the vascular mechanism in acrocyanosis. Heart, 1930, $15,229$.

10. Landis, E. M., Micro-injection studies of capillary blood pressure in Raynaud's disease. Heart, 1930, 15, 247. 